\title{
A Statistical Motion Model Based on Biomechanical Simulations for Data Fusion during Image-Guided Prostate Interventions
}

\author{
Yipeng $\mathrm{Hu}^{1}$, Dominic Morgan ${ }^{1}$, Hashim Uddin Ahmed $^{2}$, Doug Pendsé ${ }^{3,4}$, \\ Mahua Sahu ${ }^{3}$, Clare Allen ${ }^{4}$, Mark Emberton ${ }^{2}$, David Hawkes ${ }^{1}$, and Dean Barratt ${ }^{1}$ \\ ${ }^{1}$ Centre for Medical Image Computing, University College London, London, UK \\ ${ }^{2}$ Department of Urology, University College London, London, UK \\ ${ }^{3}$ National Medical Laser Centre, University College London, London, UK \\ ${ }^{4}$ Department of Radiology, University College Hospital, London, UK
}

\begin{abstract}
A method is described for generating a patient-specific, statistical motion model (SMM) of the prostate gland. Finite element analysis (FEA) is used to simulate the motion of the gland using an ultrasound-based 3D FE model over a range of plausible boundary conditions and soft-tissue properties. By applying principal component analysis to the displacements of the FE mesh node points inside the gland, the simulated deformations are then used as training data to construct the SMM. The SMM is used to both predict the displacement field over the whole gland and constrain a deformable surface registration algorithm, given only a small number of target points on the surface of the deformed gland. Using 3D transrectal ultrasound images of the prostates of five patients, acquired before and after imposing a physical deformation, to evaluate the accuracy of predicted landmark displacements, the mean target registration error was found to be less than $1.9 \mathrm{~mm}$.
\end{abstract}

Keywords: Transrectal ultrasound, minimally-invasive interventions, prostate, statistical shape modelling, biomechanical modelling.

\section{Introduction}

Transrectal ultrasound (TRUS) is used routinely to guide needle biopsy and therapeutic interventions for prostate cancer. However, TRUS images typically provide only very limited information on the spatial location of tumours. Therefore, the ability to fuse additional information on tumour location, derived from magnetic resonance (MR) imaging as well as needle and novel optical biopsy techniques, with TRUS images represents a major step towards improving the accuracy of image-guided interventions for prostate cancer. In particular, as the sensitivity and specificity of functional and structural MR imaging techniques for detecting and localising prostate cancer continue to improve, and new molecular imaging methods become available, these techniques potentially provide important information for targeting biopsy sampling and therapy delivery. Furthermore, as new minimally-invasive therapies, such as cryotherapy and high-intensity focused US (HIFU), gain popularity, there is an increasing clinical demand for 
technology that enables preoperative data to be used to deliver therapy at a specific location in order to spare adjacent tissue and minimise the risk of side effects.

Unfortunately, significant gland motion (including deformation) can occur between different image acquisitions due to forces exerted by the bladder and rectum, different patient positions, and the insertion of an endorectal US probe or MR coil [1]. Large changes in gland volume also commonly occur during and following interventions as a physiological response to needle insertion, ablative therapy, or both. In general, most conventional (geometrically constrained) image registration techniques perform poorly when significant organ motion is present due to physically implausible deformations being allowed. Consequently, there is a great deal of interest in motion modelling techniques to help constrain standard algorithms so that the output deformation fields are physically plausible. Statistical models have been reported for describing prostate deformation, which are based on training data drawn from a sample population of patient images [2]. However, establishing anatomical correspondence, both between and within patients, and analysing a sufficiently large training dataset to accurately estimate the variance in both shape and deformation, are challenging tasks. Furthermore, modelling methods based purely on the statistics of shape and deformation can be highly inaccurate when applied to an "atypical" gland that has a shape (or other properties) not represented adequately in the training population.

Some of the limitations highlighted above may be overcome by adopting physically based, biomechanical modeling techniques, such as finite element analysis (FEA), to predict motion given a 3D model of the prostate gland and surrounding tissue [3]-[5]. However, the accuracy of displacements predicted using this approach normally depends on the accuracy of boundary conditions (such as the position and orientation of the TRUS probe) and the values assigned to the elastic properties of soft tissues, which are known to vary considerably between patients: Chi et al., for example, report a registration error of up to $4.5 \mathrm{~mm}$ due solely to a $30 \%$ uncertainty in material properties for a solid FE model of the prostate [5]. Furthermore, the computation time of FEA increases with the complexity of the model, which can make this method impractical in the interventional situation due to clinical time constraints, even when, for example, graphical processor units are employed.

In this paper, we adopt a combined biomechanical-statistical approach similar to that proposed to that by Mohamed et al. [6] in which a statistical deformation model is constructed from simulated deformations of a FE model of the prostate gland under different boundary conditions (TRUS probe position). However, we extend this approach to include material properties as variable parameters in the biomechanical simulations and validate the accuracy of a deformable registration algorithm, which uses information on gland deformation provided by the statistical model. This approach addresses the problem of uncertainty in material properties associated with direct FEA by using a statistical approach to capture variability in these parameters. Furthermore, since a SMM is inherently compact, it can be used to compute 3D solid models of the deformed prostate very rapidly, and is therefore well suited to intraoperative use. Alterovitz et al. [7] describe a method for estimating prostate deformation in 2D using FEA of a model based on MR images, which includes material properties and external forces as unknown parameters in the estimation algorithm. However, to the best of the Authors' knowledge, the present study is the first to report on the application and validation of full 3D FEA to estimate gland motion in a statistical 
framework without requiring accurate knowledge of material properties or the position of the TRUS probe and pelvic bone relative to the prostate.

\section{Methods}

A schematic overview of the registration scheme developed in this work is shown in Fig. 1. The steps involved can be summarised as follows:

a) Build a patient-specific 3D FE model based on a manually segmented 3D TRUS image (i.e., source image). In this work, the prostate gland was segmented using manual contouring, but a more automated method would be highly desirable.

b) Perform a large number of FEA simulations using a range of boundary conditions (specifically, the position of the TRUS probe/balloon and position and size of the pelvis relative to the prostate gland) and range of different material properties assigned to homogeneous regions of the FE model.

c) Apply principal component analysis (PCA) on the predicted displacement of FE mesh node points and build a SMM using the principal modes of variation in node displacement.

d) Identify a small number of surface points in planes corresponding to 6 slices through a 3D TRUS image of the deformed gland (i.e., the target image).

e) Register the deformable gland model to the target surface points by optimising the weights of the principal modes of variation of the SMM such that the distance between the target point set and the deformed model surface is minimised.

To enable rigorous validation without using implanted fiducial markers, in this study both the target and source TRUS images were acquired at the start of a procedure. However, it is intended that, in clinical practice, the source image would be acquired prior to a procedure, using either 3D TRUS or MR. Stages a-c are computationally intensive and would be performed before the intervention, whereas stages $d$ and e would take place during an intervention (in realtime). Details of the experimental methods used in this study are provided in the following sections.

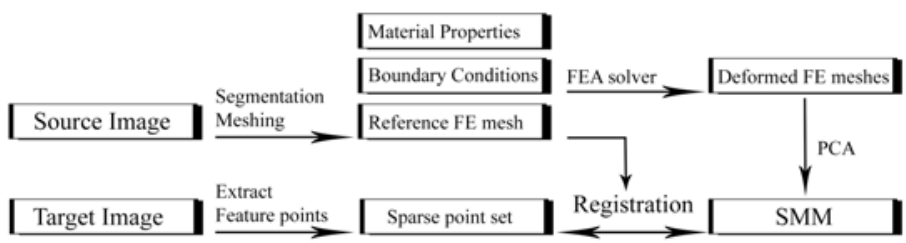

Fig. 1. Schematic overview of the method adopted. In this study, the source and target images were 3D TRUS images obtained before and after expanding the saline-filled balloon around the TRUS probe, respectively.

\subsection{D TRUS Volume Acquisition}

Three-dimensional TRUS images of the prostate were acquired for 5 patients undergoing a template-guided biopsy, or HIFU or photodynamic therapy (PDT) for treatment of prostate cancer. All patients were recruited to clinical research studies, 
approved by the local research ethics committee at University College Hospital, and gave written consent to participate. In the case of biopsy and PDT, a set of parallel transverse B-mode US images was obtained using a B-K ProFocus scanner (B-K Medical, Berkshire, UK) and a mechanical stepper mechanism (Tayman Medical Inc., MO, USA) to translate the probe (B-K $8658 \mathrm{~T}, 5-7.5 \mathrm{MHz}$ transducer) axially along the rectum. Images were captured at $2 \mathrm{~mm}$ intervals and stored on the US scanner. In the case of HIFU therapy, 3D volumes were acquired automatically using a Sonablate ${ }^{\circ}$ 500 system (Focus Surgery, Inc., Indiana, USA). Two volumes were acquired for each patient at the start of the procedure: one with the balloon at minimal expansion, and the other after expanding the balloon by injecting saline with a syringe in order to deform the prostate gland. Expanding the balloon in this way simulates the motion of the prostate gland that might typically occur due to the presence of a TRUS probe or an endorectal MR imaging coil. The first volume was chosen as the source image for building the SMM, whilst the second was used for accuracy evaluation.

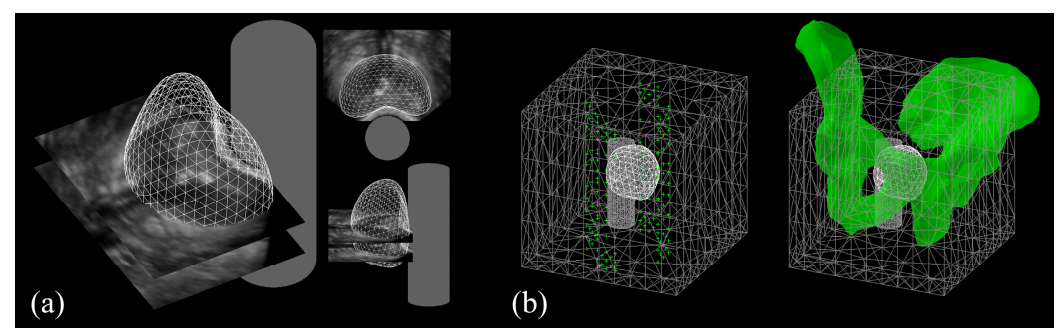

Fig. 2. (a) Three views of the triangulated surface mesh and cylinder representing the rectal balloon surface. (b) Illustration of the two methods used to constrain displacements away from the prostate: left - simple constraint (fixed node points are shown in green); right - realistic pelvis-shaped constraint (shown in green).

\subsection{Finite Element Model Construction}

In this study, the prostate was segmented from each 3D TRUS volume by manually contouring the prostate capsule on each acquired transverse slices. The gland was further segmented into an inner and outer gland, based on differences in echotexture on the US images. A two material model was chosen for the prostate because of the significantly different material properties between these regions [3]. A smooth surface was fitted to the contours points using a spherical harmonic representation from which a triangulated mesh was generated (see Fig. 2a). The surface of the balloon in contact with the rectum was modelled as a cylinder. The diameter and position of the cylinder was determined automatically by performing a least squares fit to points on the balloon surface, extracted from the TRUS images using Canny edge detection. The tissue surrounding the prostate was modelled as a homogeneous block with dimensions $20 \times 20 \times 20 \mathrm{~cm}^{3}$. The surface meshes and the block structure were imported into the commercial FEA software ANSYS (ANSYS Europe Ltd., Oxfordshire, UK) using the solid modelling tool. The FE model was then constructed and meshed into 30-40,000 tetrahedral elements using trimmed parametric surfaces and Delaunay tessellation techniques provided by the software. Ten-node tetrahedral elements were used as these support non-linear geometries using unstructured meshes. Regions 
corresponding to the each part of the prostate gland, the rectal wall, and surrounding tissue were labelled separately. All tissues were assumed to behave as isotropic, linear elastic materials under loading, and node displacements were computed using the preconditions conjugate gradient iterative equation solver in ANSYS.

\subsection{Boundary Conditions}

FEA simulations of prostate motion were performed using the three different sets of boundary conditions are summarised in Table 1 . These are explained as follows:

Table 1. Summary of boundary condition configurations used for FEA simulations

\begin{tabular}{cllc}
\hline SMM & Balloon position & Bony constraints & Degrees of freedom \\
\hline M1 & Fixed & Fixed - Simple constraint & 1 \\
M2 & Fixed & Variable pelvis position and size & 5 \\
M3 & Variable & Variable pelvis position and size & 8 \\
\hline
\end{tabular}

Balloon/TRUS probe displacement: The expansion of the balloon was modelled by displacing the cylinder surface nodes radially to lie on the surface of an enlarged cylinder fitted to balloon surface points detected in the target (i.e., deformed) TRUS image. This step provided a fixed boundary condition for the FEA simulations, which could be easily and automatically performed during an intervention. However, as the assumption of no probe/patient motion may not always be valid, a random displacement in the range -5.0 to $5.0 \mathrm{~mm}$ was added to each of the $x, y$ and $z$ co-ordinates of the cylinder to compare the performance of the resulting SMMs.

Bony constraints: Because only a very small part of the pelvic bone is visualised in TRUS images, it was not possible to accurately estimate its location relative to the prostate. Two methods for introducing bony constraints into the FE model were used, as shown in Fig. 2b. In the first, mesh nodes lying on four-strips around the gland were fixed. In the second, the nodes on the surface of a realistically-shaped model of the pelvic bone were fixed. The pelvis model was generated by a statistical shape model derived from CT images [8]. The initial size of the bone was set to the mean and the initial position was determined using the method described in [9]. In two of the series of biomechanical simulations the position and size of the pelvis were varied by adding a random (normally distributed) displacement of $0.0 \pm 5.0 \mathrm{~mm}$ (mean $\pm \mathrm{SD}$ ) to the $\mathrm{x}-, \mathrm{y}-$, and $\mathrm{z}$-components of the pelvis position vector, and by varying a global scaling factor with a mean of 1.0 and a SD of 0.2 .

\subsection{Material Properties}

Since it can been argued that the assumption of incompressibility (Poisson's ratio, $v=0.5$ ) may not be appropriate for the prostate (and perhaps other soft-tissue) because of fluid gain and loss, and the presence of a collapsible urethra, both the Young's modulus $(E)$ and the Poisson's ratio ( $v$ ) assigned to different materials in the FE model were assumed to be unknown. Therefore, the relative values of these parameters were varied for the prostate compartments and surrounding tissue block in the FEA simulations. The values assigned 
to $E$ and $v$ for the inner and outer prostate gland and the surrounding tissue were sampled at 4 uniformly-spaced intervals over the ranges $10-200 \mathrm{kPa}$ and $0.01-0.49$, respectively. The rectal wall in contact with the balloon was however assumed to be nearly incompressible as it is thin and already compressed, and was assigned the fixed values $E=100 \mathrm{kPa}$ and $\nu=0.49$, which had the effect of reducing the number of degrees of freedom in the simulations to 6 . Subsequently, $4^{6}=4096$ simulations were performed for each of the 3 boundary condition configurations given in Table 1 .

\subsection{Statistical Motion Model}

For each of $M$ (=4096) simulated gland deformations, the 3D displacement of every node in the prostate gland mesh was calculated and combined to form a $3 N \times 1$ vector, $\mathbf{x}$, which describes the predicted displacement of the gland mesh nodes $(N$ is the number of nodes). The principal modes of variation in $\mathbf{x}$ were then calculated using PCA. If $\mathbf{S}_{0}$ is a vector containing the co-ordinates of the node points of the initial model, the vector, $\mathbf{S}$, containing the corresponding node positions of a deformed prostate is given by:

$$
\mathbf{S}=\mathbf{S}_{0}+\overline{\mathbf{x}}+\sum_{i=1}^{L} c_{i} \mathbf{e}_{i}, \quad 1 \leq L \leq M,
$$

where $\overline{\mathbf{X}}$ is the mean displacement vector, $\mathbf{e}_{i}$ is the $i^{\text {th }}$ eigenvector of the covariance matrix, and $c_{i}$ is a scalar weight. $L$ was chosen so that the SMM covered $>99 \%$ of variance in the training data and gave a model reconstruction error, $\varepsilon,<0.1 \mathrm{~mm}$, where

$$
\mathcal{E}=\sqrt{\frac{1}{3 N}\left(\sum_{i=1}^{M} c_{i} \mathbf{e}_{i}-\sum_{i=1}^{L} c_{i} \mathbf{e}_{i}\right)^{\mathrm{T}}\left(\sum_{i=1}^{M} c_{i} \mathbf{e}_{i}-\sum_{i=1}^{L} c_{i} \mathbf{e}_{i}\right)} .
$$

\subsection{Model Registration}

In practice, a full segmentation of the prostate surface in the target TRUS image is impractical during a surgical procedure because manual contouring remains the most reliable and accurate way of delineating the capsule. However, since the resulting SMM is very well constrained, only a sparse set of surface points is required to register the deformable prostate model. To simulate a simple and clinically feasible protocol for defining target points, the segmented target TRUS image was resliced in 3 sagittal and 3 transverse planes and 6 evenly-spaced points computed along the surface contours in each slice. Starting with the mean shape, the first $L$ weights, $\left\{c_{1}\right.$, $\left.c_{2}, \ldots, c_{L}\right\}$, were optimised to minimise the distance between the target points and the deformed model surface in MATLAB using a nonlinear least-squares optimisation algorithm. Once registered, the positions of any point inside the deformed gland can be calculated by interpolating the node point positions. For the purposes of comparison with a rigid registration algorithm, the source (i.e., undeformed) surface was registered to the target points using the well-known ICP algorithm.

\subsection{Accuracy Validation}

A number of corresponding landmarks, including cysts, calcifications and the urethra, were identified manually in the source and target TRUS volumes. The landmarks in 


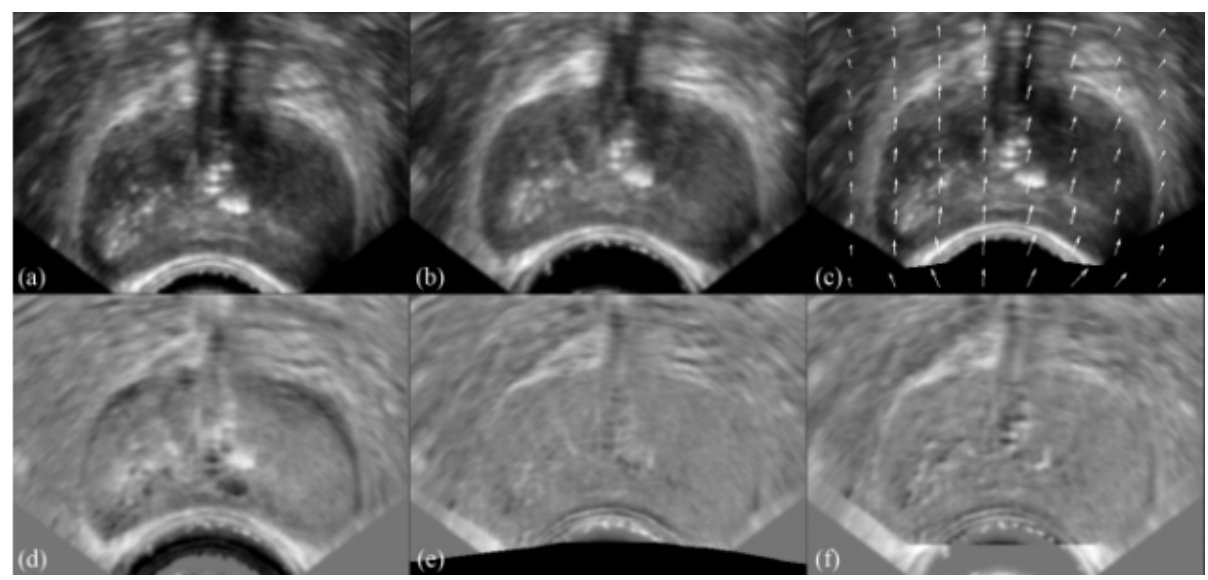

Fig. 3. Example transverse slices through 3D TRUS images (Case 1). From top left: (a) Source image (balloon minimally expanded); (b) Target image (balloon maximally expanded); (c) Source image after warping using the interpolated displacement field generated by registering the SMM (shown as white arrows); (d)-(f) Subtracted images: (d) target - source (b-a); (e) target - warped source $(b-c)$; (f) target - source after rigid registration.

Table 2. Target registration errors calculated for anatomical landmarks (the boundary conditions used for the models M1, M2 and M3 are summarised in Table 1)

\begin{tabular}{lcccccc}
\hline Case No. & No. & \multicolumn{2}{c}{ Mean \pm SD TRE (mm) } \\
& Landmarks & Start & Rigid & M1 & M2 & M3 \\
\hline 1 (Biopsy) & 12 & $5.45 \pm 1.16$ & $3.32 \pm 0.98$ & $1.90 \pm 0.54$ & $1.83 \pm 0.61$ & $2.04 \pm 0.61$ \\
2 (PDT) & 7 & $4.32 \pm 1.39$ & $2.59 \pm 0.50$ & $1.45 \pm 0.96$ & $1.45 \pm 0.92$ & $2.06 \pm 0.76$ \\
3 (HIFU) & 8 & $5.03 \pm 0.78$ & $2.23 \pm 1.28$ & $1.77 \pm 0.54$ & $1.86 \pm 0.52$ & $2.05 \pm 0.67$ \\
4 (Biopsy) & 15 & $4.47 \pm 1.02$ & $2.63 \pm 0.77$ & $1.86 \pm 0.52$ & $1.80 \pm 0.51$ & $1.49 \pm 0.68$ \\
5 (HIFU) & 6 & $6.42 \pm 1.48$ & $3.73 \pm 0.98$ & $2.38 \pm 0.86$ & $2.32 \pm 0.86$ & $2.22 \pm 0.76$ \\
\hline All & 48 & $5.03 \pm 1.29$ & $2.87 \pm 1.01$ & $1.86 \pm 0.67$ & $1.83 \pm 0.67$ & $1.89 \pm 0.71$ \\
\hline
\end{tabular}

the source image were then propagated into the target image space using the displacement field produced by the SMM. For each pair of landmarks, a target registration error (TRE) was calculated, defined as the distance between the manually defined position and the propagated position of a landmark in the target image space.

\section{Results}

The landmark-based TREs are given in Table 2. Fig. 3 illustrates the difference between transverse TRUS slices after deformable versus rigid registration for Case 1 . All registrations were completed within 10 s on a PC with a $2.33 \mathrm{GHz}$ Intel ${ }^{\circledR}$ Core $^{\mathrm{TM}}$ dual CPU processor and 3GB of RAM (between 7 and 11 principal modes were used for the SMMs). The average time taken to compute each SMM was approximately 50 hours. Inspection of the results in Table 2 reveals that the most accurate registrations were achieved using an SMM to constrain the deformation. Although a significant proportion of gland motion $(\sim 40 \%)$ is recovered using a rigid registration scheme, the 
SMM-constrained deformable registration on average recovered approximately $60 \%$ of the motion, depending on the amount of deformation. An interesting observation is that including bony constraints and associated additional degrees of freedom in the SMM relating to the position of the pelvic bone made very little difference to the overall registration accuracy inside the gland.

\section{Discussion}

The approach in this study combines statistical shape modelling techniques with biomechanical simulations to generate a patient-specific, 3D deformable model that can be rapidly registered to a small number of surface points identified from TRUS slices. Since the model can be generated before a procedure and does not require accurate estimates of material properties, this method is clinically feasible, although further work is required to reduce the amount of pre-procedural computation and manual interaction, and to investigate the accuracy of generating an SMM from other imaging modalities, such as MR. In this case, the diameter and pose of the TRUS balloon are unknown a priori and therefore some additional degrees of freedom would be required (although good estimates could easily be obtained, particularly if a standardised protocol is adopted for probe insertion). The results of this study suggest that a complex FE model with accurate bony constraints derived from MR or CT data may be unnecessary when only the motion of the prostate is of interest. The use of TRUS data made it much easier to determine an accurate gold standard for landmark displacements compared with multimodal images, since identifying multiple corresponding intra-gland landmarks is difficult without using fiducial markers.

\section{References}

1. Byrne, T.E.: A review of prostate motion with considerations for the treatment of prostate cancer. Medical Dosimetry 30, 155-161 (2005)

2. Dam, E.B., et al.: Prostate shape modelling based on principal geodesic analysis bootstrapping. In: Barillot, C., Haynor, D.R., Hellier, P. (eds.) MICCAI 2004. LNCS, vol. 3217, pp. 1008-1016. Springer, Heidelberg (2004)

3. Bharatha, A., et al.: Evaluation of three-dimensional finite element-based deformable registration of pre-and intraoperative prostate imaging. Med. Phys. 28, 2551-2560 (2001)

4. Crouch, J.R., et al.: Automated finite element analysis for deformable registration of prostate images. IEEE Trans. on Med. Imag. 26, 1379-1390 (2007)

5. Chi, Y., et al.: A material sensitivity study on the accuracy of deformable organ registration using linear biomechanical models. Med. Phys. 33, 421-433 (2006)

6. Mohamed, A., et al.: A combined statistical and biomechanical model for estimation of intra-operative prostate deformation. In: Dohi, T., Kikinis, R. (eds.) MICCAI 2002. LNCS, vol. 2489, pp. 452-460. Springer, Heidelberg (2002)

7. Alterovitz, R., et al.: Registration of MR prostate images with biomechanical modeling and nonlinear parameter estimation. Med. Phys. 33, 446-454 (2006)

8. Thompson, et al.: Use of a CT statistical deformation model for multi-modal pelvic bone segmentation. In: Proc. SPIE Medical Imaging (2008)

9. Schallenkamp, J., et al.: Prostate position relative to pelvic bony anatomy based on intraprostatic gold markers and electronic portal imaging. Int. J. Radiation Oncology Biol. Phys. 63, 800-811 (2005) 